\title{
An Explicit Construction of a sequence of codes attaining the Vladut-Zink Bound:The first steps
}

\author{
Høholdt, Tom; Voss, Cornelia
}

Published in:

I E E E Transactions on Information Theory

Link to article, DOI:

$10.1109 / 18.567659$

Publication date:

1997

Document Version

Publisher's PDF, also known as Version of record

Link back to DTU Orbit

Citation (APA):

Høholdt, T., \& Voss, C. (1997). An Explicit Construction of a sequence of codes attaining the Tsfasman-VladutZink Bound:The first steps. I E E E Transactions on Information Theory, 43(1), 128-135.

https://doi.org/10.1109/18.567659

\section{General rights}

Copyright and moral rights for the publications made accessible in the public portal are retained by the authors and/or other copyright owners and it is a condition of accessing publications that users recognise and abide by the legal requirements associated with these rights.

- Users may download and print one copy of any publication from the public portal for the purpose of private study or research

- You may not further distribute the material or use it for any profit-making activity or commercial gain

- You may freely distribute the URL identifying the publication in the public portal

If you believe that this document breaches copyright please contact us providing details, and we will remove access to the work immediately and investigate your claim 


\title{
An Explicit Construction of a Sequence of Codes Attaining the Tsfasman-Vlădut-Zink Bound The First Steps
}

\author{
Conny Voss and Tom Høholdt, Member, IEEE
}

\begin{abstract}
We present a sequence of codes attaining the Tsfasman-Vlădut-Zink bound. The construction is based on the tower of Artin-Schreier extensions recently described by Garcia and Stichtenoth. We also determine the dual codes. The first steps of the constructions are explicitely given as generator matrices.
\end{abstract}

Index Terms-Algebraic geometric codes, asymptotically good codes.

\section{INTRODUCTION}

L ET $\mathbb{F}_{l}$ be the finite field of cardinality $l$ and let $\left(F_{i}\right)_{i \geq 1}$ be a sequence of algebraic function fields over $\mathbb{F}_{l}$ where $F_{i} / F_{l}$ has genus $g_{i}$ and $N_{i}=N\left(F_{i}\right)$ places of degree one such that $g_{i} \rightarrow \infty$ and

$$
\lim _{i \rightarrow \infty} N_{i} / g_{i}>1
$$

It is well known (see [6], [8]) that in this situation one can construct asymptotically good sequences of algebraic geometric (geometric Goppa) codes over $\mathbb{F}_{l}$.

Let $N_{l}(g):=\max \{N(F) \mid F$. is a function field of genus $g$ over $\left.\mathbb{F}_{l}\right\}$ and

$$
A(l):=\lim _{g \rightarrow \infty} \sup _{l} N_{l}(g) / g .
$$

The Drinfeld-Vlădut bound (see [1]) tells us that

$$
A(l) \leq \sqrt{l}-1
$$

and it was shown by Ihara [3] and Tsfasman, Vlădut, and Zink [7] that, if $l=q^{2}$ is a square

$$
A\left(q^{2}\right)=q-1
$$

For $l$ a square, $l \geq 49$ and $\lim _{i \rightarrow \infty} N_{i} / g_{i}=A(l)$ the Tsfasman-Vlădut-Zink (TVZ) theorem [7] says that the parameters of the related algebraic geometric codes are better than the Gilbert-Varshamov bound in a certain range of the rate. In [4] and [9] it is shown how to reach the TVZ bound with a polynomial construction but the complexity of this algorithm is so high that the actual construction, i.e., generator or parity-check matrices of the code, is intractable. In a recent preprint by Feng and Rao [10], the authors claimed to have

Manuscript received May 24, 1995; revised March 15, 1996. The material in this paper was presented in part at the AGCT-5, Luminy, France, June 1996.

The authors are with the Department of Mathematics, Technical University of Denmark, Bldg. 303, DK-2800 Lyngby, Denmark.

Publisher Item Identifier S 0018-9448(97)00158-2. found asymptotically good codes in an elementary way using socalled generalized Klein curves which are defined by the equations

$$
x_{i+1}^{3} x_{i}+x_{i}^{3}+x_{i+1}=0, i=1, \cdots, m-1
$$

over GF (8). Pellikaan tried to figure out whether their claim was correct (the curves are asymptotically bad as recently found out by Garcia and Stichtenoth) and suggested the curves with equations

$$
x_{i+1}^{2} x_{i}+x_{i}^{2}+x_{i+1}=0, \quad i=1, \cdots, m-1 \text { over } \mathrm{GF}(4) .
$$

It turned out that this gave a tower of Artin-Schreier extensions which enabled Garcia and Stichtenoth to generalize to an arbitrary square power $q$ and to calculate the genera and the number of $\mathbb{F}_{q}$-rational points and therefore to prove that the curves were asymptotically good, so we have a tower of function fields $\left(F_{i}\right)_{i \geq 1}$ over $\mathbb{F}_{q^{2}}$ reaching the Drinfeld-Vlădut bound $A\left(q^{2}\right)=q-1$. The function fields of this tower are defined in the following way:

Definition 1.1: Let $F_{1}:=\mathbb{F}_{q^{2}}\left(x_{1}\right)$ be the rational function field over $\mathbb{F}_{q^{2}}$. For $n \geq 1$ let

$$
F_{n+1}:=F_{n}\left(z_{n+1}\right)
$$

where $z_{n+1}$ satisfies the equation

$$
z_{n+1}^{q}+z_{n+1}=x_{n}^{q+1}
$$

with

$$
x_{n}:=z_{n} / x_{n-1} \quad(\text { for } n \geq 2) .
$$

In this paper we first present sequences of asymptotically good algebraic geometric codes related to the function field tower of Garcia and Stichtenoth, and we determine their dual codes as well.

For a function field $F_{i} / \mathbb{F}_{q^{2}}$ an algebraic geometric code $C_{i}$ is of the form $C_{i}=C_{\mathcal{L}}\left(D_{i}, G_{i}\right)$ with $D_{i}=P_{1}+\cdots+P_{n}$ where the $P_{j}$ 's are pairwise-distinct places of degree one in $F_{i} / \mathbb{F}_{q^{2}}$, and $G_{i}$ a divisor of $F_{i} / \mathbb{F}_{q^{2}}$ such that $\operatorname{supp}\left(G_{i}\right) \cap \operatorname{supp}\left(D_{i}\right)=$ $\emptyset$. Then

$$
C_{i}=\left\{\left(f\left(P_{1}\right), \cdots, f\left(P_{n}\right)\right) \mid f \in \mathcal{L}\left(G_{i}\right)\right\} \subseteq\left(\mathbb{F}_{q^{2}}\right)^{n} .
$$

For applications of such codes in practice one needs an explicit description, which means an explicit basis for the vector space $\mathcal{L}\left(G_{i}\right)$ or a generator matrix of the code $C_{i}$. 


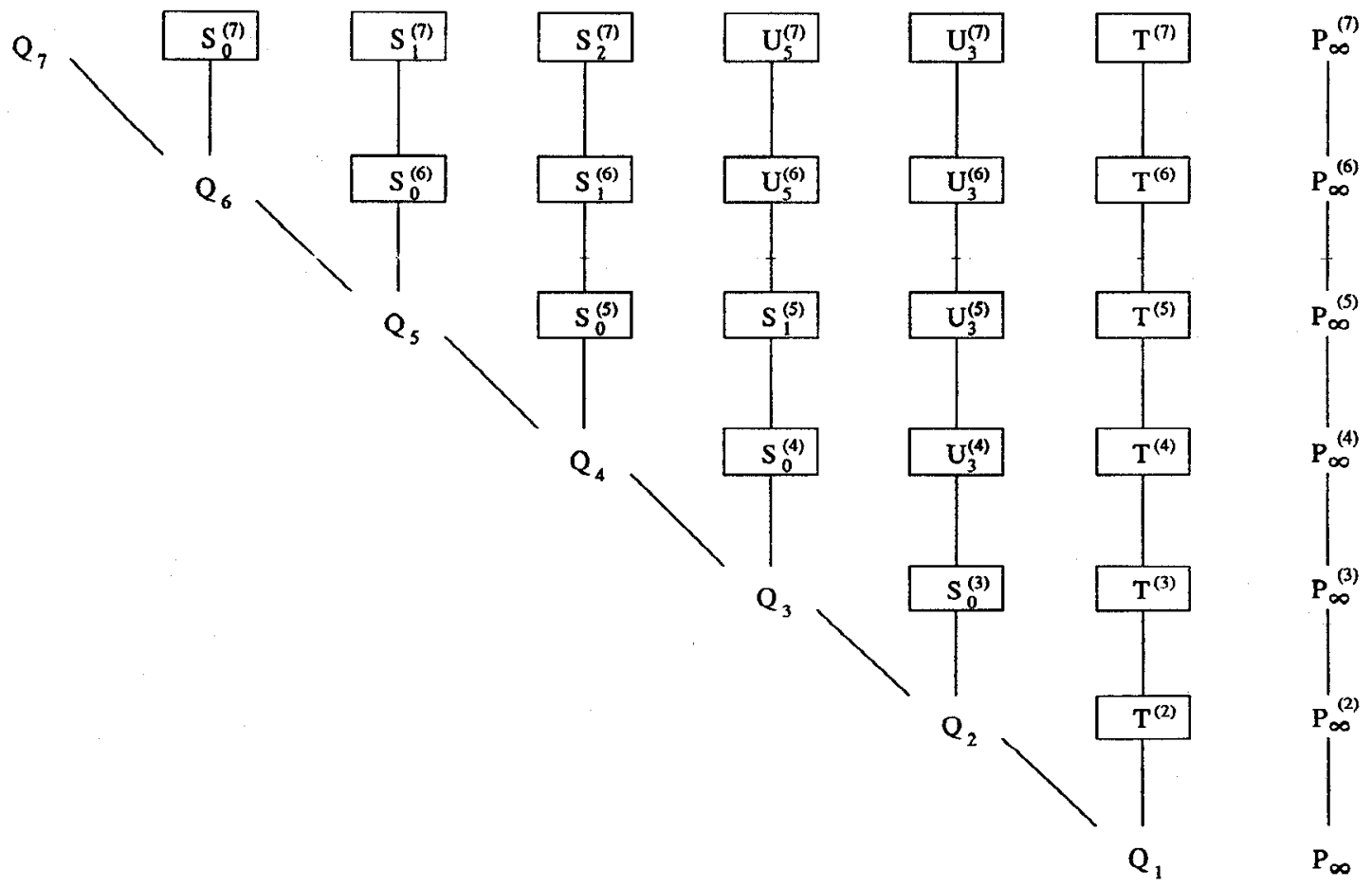

Fig. 1.

The second function field $F_{2}$ in the tower is the Hermitian function field and the related codes $C_{2}$ in our sequences are the well-known Hermitian codes (see, e.g., [6]). In the second part of this paper we will describe the codes $C_{3}$ corresponding to $F_{3}$ in detail by constructing a basis of $\mathcal{L}\left(G_{3}\right)$ and a generator matrix for $C_{3}$. As in the Hermitian case, it turns out that the dual codes of the codes $C_{3}$ are of the same type.

From the special case $G_{3}=s P_{\infty}$, where $s>0$ and $P_{\infty}$ is the pole of $x_{1}$ in $F_{3}$, we get the pole numbers of $P_{\infty}$. While in $F_{2}$ the pole numbers of the pole of $x_{1}$ are generated by only two numbers, namely, $q$ and $q+1$; it turns out that in $F_{3}$ one in general needs more than three numbers to generate the whole set of pole numbers.

Our bases for the vector spaces $\mathcal{L}\left(G_{3}\right)$ consist of monomial expressions in $x_{1}, x_{2}$, and $z_{3}$ (where negative exponents are possible) which makes it easy to give a generator matrix for the codes $C_{3}$. One could maybe hope that, in a similar manner, a general description of the spaces $\mathcal{L}\left(G_{i}\right)$ for $i \geq 1$ would be possible, but unfortunately already for $G_{4}$ monomial expressions in $x_{1}, x_{2}, x_{3}$, and $z_{4}$ are not sufficient to generate the whole space.

\section{PRELIMINARIES}

We start with some notation and definitions that are used throughout this paper. Many of them are the same as in [2].

\footnotetext{
$F_{i}, i \geq 1 \quad$ function fields as defined in Definition 1.1; $g_{i}=g\left(F_{i}\right) \quad$ genus of $F_{i} / \mathbb{F}_{q^{2}}$;

$\mathbb{P}(F) \quad$ set of places of the function field $F / \mathbb{F}_{\mathrm{q}^{2}}$;

$N_{i}=N\left(F_{i}\right)$ number of places $P \in \mathbb{P}\left(F_{i}\right)$ of degree one;

$v_{p} \quad$ normalized discrete valuation associated with $P$
}

Diff $\left(F_{n} / F_{k}\right)$ different of the extension $F_{n} / F_{k}(k<n)$;
$\operatorname{con}_{F_{n} / F_{k}}(A)$ conorm of a divisor $A$ of $F_{k}$ in $F_{n}(k<n)$; $P \cap F_{k} \quad$ restriction of a place $P \in \mathbb{P}\left(F_{n}\right)$ to $F_{k}(n>k)$.

We recall some properties of the function fields $F_{i} / \mathbb{F}_{q^{2}}$ (see $[2$, Lemmas 2.1, 2.2]).

Lemma 2.1:

i) Suppose that a place $P \in \mathbb{P}\left(F_{n}\right)$ is a simple pole of $x_{n}$ in $F_{n}$. Then the extension $F_{n+1} / F_{n}$ has degree $\left[F_{n+1}: F_{n}\right]=q$ and $P$ is totally ramified in $F_{n+1} / F_{n}$. The place $P^{\prime} \in \mathbb{P}\left(F_{n+1}\right)$ lying above $P$ is a simple pole of $x_{n+1}$.

ii) For all $n \geq 1$ there is a unique place $Q_{n} \in \mathbb{P}\left(F_{n}\right)$ which is a common zero of the functions $x_{1}, z_{2}, z_{3}, \cdots, z_{n}$. Its degree is $\operatorname{deg} Q_{n}=1$. For $1 \leq k \leq n$, the place $Q_{n}$ is also a zero of $x_{k}$, and we have $v_{Q_{n}}\left(x_{k}\right)=q^{k-1}$. In the extension $F_{n+1} / F_{n}$ the place $Q_{n}$ splits into $q$ places of $F_{n+1}$ of degree one (one of them being $Q_{n+1}$ ).

We introduce the following sets of places and divisors: Definition 2.2: See Fig. 1.

i) For $n \geq 3$, let

$$
S_{0}^{(n)}:=\left\{P \in \mathbb{P}\left(F_{n}\right) \mid P \cap F_{n-1}=Q_{n-1} \text { and } P \neq Q_{n}\right\}
$$

and

$$
D_{0}^{(n)}:=\sum_{P \in S_{0}^{(n)}} P
$$

ii) For $1 \leq i \leq\lfloor(n-3) / 2\rfloor$, let

$$
S_{i}^{(n)}:=\left\{P \in \mathbb{P}\left(F_{n}\right) \mid P \cap F_{n-1} \in S_{i-1}^{(n-1)}\right\}
$$


and

$$
D_{i}^{(n)}:=\sum_{P \in S_{i}^{(n)}} P
$$

iii) Let

$$
T^{(2)}:=\left\{P \in \mathbb{P}\left(F_{2}\right) \mid P \cap F_{1}=Q_{1} \text { and } P \neq Q_{2}\right\}
$$

and

$$
E^{(2)}:=\sum_{P \in T^{(2)}} P
$$

and for $n \geq 3$, let

$$
T^{(n)}:=\left\{P \in \mathbb{P}\left(F_{n}\right) \mid P \cap F_{n-1} \in T^{(n-1)}\right\}
$$

and

$$
E^{(n)}:=\sum_{P \in T^{(n)}} P
$$

iv) For $n \geq 4$ and $n \equiv 0 \bmod 2$, let

$$
U_{n-1}^{(n)}:=\left\{P \in \mathbb{P}\left(F_{n}\right) \mid P \cap F_{n-1} \in S_{(n-4) / 2}^{(n-1)}\right\}
$$

and

$$
M_{n-1}^{(n)}:=\sum_{P \in U_{n-1}^{(n)}} P
$$

and for $n \geq 5$ and $1 \leq i \leq\lfloor(n-2) / 2\rfloor$, let

$$
U_{2 i+1}^{(n)}:=\left\{P \in \mathbb{P}\left(F_{n}\right) \mid P \cap F_{n-1} \in U_{2 i+1}^{(n-1)}\right\}
$$

and

$$
M_{2 i+1}^{(n)}:=\sum_{P \in U_{2 i+1}^{(n)}} P .
$$

v) Let $P_{\infty} \in \mathbb{P}\left(F_{1}\right)$ denote the pole of $x_{1}$ in $F_{1}$ and for $n \geq 2$, let $P_{\infty}^{(n)}$ be the unique extension of $P_{\infty}$ in $F_{n}$.

\section{SEQuenCES OF Asymptotically GOOD CODES AND THEIR DUALS}

Definition 3.1: For $\alpha \in \mathbb{F}_{q^{2}}$ we denote the zero of $x_{1}-\alpha$ in $F_{1}$ by $P_{\alpha}$. We define

$$
\begin{aligned}
& D^{(1)}:=\sum_{\alpha \in \mathbb{F}_{q^{2}}} P_{\alpha} \\
& G^{(1)}:=s P_{\infty} \\
& D^{(2)}:=Q_{2}+E^{(2)}+\sum_{\alpha \in F_{q^{2}} \backslash\{0\}} \operatorname{con}_{F_{2} / F_{1}}\left(P_{\alpha}\right) \\
& G^{(2)}:=s P_{\infty}^{(2)} \text { with } 0 \leq s \leq q^{3}+q^{2}-q-2
\end{aligned}
$$

and for $n \geq 3$

$$
\begin{aligned}
D^{(n)} & :=Q_{n}+D_{0}^{(n)}+\sum_{\alpha \in \mathrm{F}_{q^{2}} \backslash\{0\}} \operatorname{con}_{F_{n} / F_{1}}\left(P_{\alpha}\right) \\
G^{(n)} & :=\sum_{i=1}^{\lfloor(n-3) / 2\rfloor} m_{i} M_{2 i+1}^{(n)}+r E^{(n)}+s P_{\infty}^{(n)}
\end{aligned}
$$

where

$$
\begin{aligned}
& 0 \leq m_{i} \leq q^{n-2 i-1}, \quad \text { for } 1 \leq i \leq\left\lfloor\frac{n-3}{2}\right\rfloor \\
& 0 \leq r \leq q^{n-1}+q^{n-2}-q-2 \\
& 0 \leq s \leq q^{n+1}+q^{n}-q-2 .
\end{aligned}
$$

Definition 3.2: For $i \geq 1$ we define the algebraic geometric codes

$$
C_{i}:=C_{\mathcal{L}}\left(D^{(i)}, G^{(i)}\right) .
$$

Observe that the codes $C_{1}$ are generalized Reed-Solomon codes and the codes $C_{2}$ are Hermitian codes (see [6]).

For $i>3$ and $2 g_{i}-2<\operatorname{deg} G^{(i)}<\left(q^{2}-1\right) q^{i-1}+q$ the code $C_{i}$ is an $\left[n_{i}, k_{i}, d_{i}\right]$ code of length $n_{i}=\left(q^{2}-1\right) q^{i-1}+q$, dimension $k_{i}=\operatorname{deg} G^{(i)}+1-g_{i}$ and minimum distance $d_{i} \geq n_{i}-\operatorname{deg} G^{(i)}$, where

$$
\operatorname{deg} G^{(i)}=(q-1) \sum_{j=1}^{\lfloor(i-3) / 2\rfloor} m_{j} q^{j}+(q-1) r+s
$$

and (see [2, Theorem 2.10])

$$
g_{i}= \begin{cases}q^{i}+q^{i-1}-q^{(i+1) / 2}-2 q^{(i-1) / 2}+1, & \text { if } i \equiv 1 \bmod 2 \\ q^{i}+q^{i-1}-\frac{1}{2} q^{(i / 2)+1} & \text { if } i \equiv 0 \bmod 2 .\end{cases}
$$

Thus for the codes we get

$$
\frac{d_{i}}{n_{i}}+\frac{k_{i}}{n_{i}} \geq 1+\frac{1}{n_{i}}-\frac{g_{i}}{n_{i}}
$$

and the right-hand side is $1-1 / q$ in the limit as $i \rightarrow \infty$, which exactly is the Tsfasman-Vlădut-Zink bound.

In the following, we want to determine the dual codes of the codes $C_{i}, i \geq 3$. From [6, Proposition II.2.10], we know that $C_{i}^{\perp}$ is again an algebraic geometric code $C_{\mathcal{L}}\left(D^{(i)}, H^{(i)}\right)$ with

$$
H^{(i)}=D^{(i)}-G^{(i)}+\left(\eta_{i}\right)
$$

where $\eta_{i}$ is a Weil differential of $F_{i} / \mathbb{F}_{q^{2}}$ such that

$$
v_{p}\left(\eta_{i}\right)=-1 \text { and } \eta_{i_{p}}(1)=1 \text { for all } P \leq D^{(i)}
$$

( $\eta_{i_{p}}$ is the local component of $\eta_{i}$ at the place $P$ ).

In order to determine the codes $C_{i}^{\perp}$ we therefore have to find a Weil differential of $F_{i}$ with the property (3) and to determine its divisor. Since the divisor of such a differential depends on the different of $F_{i} / F_{1}$, we first compute the different.

Proposition 3.3: For $n \geq 2$ we have

$$
\begin{aligned}
\operatorname{Diff}\left(F_{n} / F_{1}\right)=(q-1)(q+2) & \left(\sum_{j=1}^{\lfloor(n-3) / 2\rfloor} \sum_{l=0}^{n-2 j-3} q^{l} M_{2 j+1}^{(n)}\right. \\
& \left.+\sum_{i=0}^{n-3} q^{i} E^{(n)}+\sum_{i=0}^{n-2} q^{i} P_{\infty}^{(n)}\right) .
\end{aligned}
$$


Proof: For $n \geq 2$ we have for the different (see [6, Corollary III.4.11])

$$
\begin{aligned}
\operatorname{Diff}\left(F_{n} / F_{1}\right)= & \operatorname{Diff}\left(F_{n} / F_{n-1}\right) \\
& +\operatorname{con}_{F_{n} / F_{n-1}}\left(\operatorname{Diff}\left(F_{n-1} / F_{1}\right)\right) .
\end{aligned}
$$

By [2], all places of $F_{n-1}$ appearing in the different of $F_{n-1}$ over $F_{1}$ are totally ramified in $F_{n}$, and $\operatorname{Diff}\left(F_{2} / F_{1}\right)=$ $(q-1)(q+2) P_{\infty}^{(2)}$ and for $n \geq 3$

$$
\operatorname{Diff}\left(F_{n} / F_{n-1}\right)=(q-1)(q+2)
$$

$$
\left(\sum_{j=1}^{\lfloor(n-3) / 2\rfloor} M_{2 j+1}^{(n)}+E^{(n)}+P_{\infty}^{(n)}\right)
$$

The proposition now follows by induction.

Next we determine the principal divisor $\left(x_{1}\right)^{(n)}$ of $x_{1}$ in $F_{n}$.

Lemma 3.4: For $n \geq 3$ we have

$$
\begin{aligned}
\left(x_{1}\right)^{(n)}= & Q_{n}+\sum_{i=0}^{\lfloor(n-3) / 2\rfloor} D_{i}^{(n)}+\sum_{i=1}^{\lfloor(n-2) / 2\rfloor} q^{n-2-2 i} M_{2 i+1}^{(n)} \\
& +q^{n-2} E^{(n)}-q^{n-1} P_{\infty}^{(n)} .
\end{aligned}
$$
get

Proof: By Lemma 2.1 and Definition 2.2 we obviously

and

$$
\left(x_{1}\right)^{(2)}=Q_{2}+E^{(2)}-q P_{\infty}^{(2)}
$$

$$
\left(x_{1}\right)^{(3)}=Q_{3}+D_{0}^{(3)}+q E^{(3)}-q^{2} P_{\infty}^{(3)} .
$$

Observing that for $n \geq 4$ and $n \equiv 0 \bmod 2$ we have $U_{n-1}^{(n)}=S_{((n-4) / 2)+1}^{(n)}$, the assertion follows immediately by induction.

Lemma 3.5: Let $z:=x_{1}^{q^{2}}-x_{1}$. Then for $n \geq 2$

i)

$$
\begin{aligned}
(z)^{(n)}= & D^{(n)}+\sum_{i=1}^{\lfloor(n-3) / 2\rfloor} D_{i}^{(n)} \\
& +\sum_{i=1}^{\lfloor(n-2) / 2\rfloor} q^{n-2 i-2} M_{2 i+1}^{(n)} \\
& +q^{n-2} E^{(n)}-q^{n+1} P_{\infty}^{(n)}
\end{aligned}
$$

ii) $(d z)^{(n)}=(q-1)(q+2)$

$$
\begin{aligned}
& \left(\sum_{j=1}^{\lfloor(n-3) / 2\rfloor} \sum_{l=0}^{n-2 j-3} q^{l} M_{2 j+1}^{(n)}+\sum_{i=0}^{n-3} q^{i} E^{(n)}\right) \\
& +\left((q-1)(q+2) \sum_{i=0}^{n-2} q^{i}-2 q^{n-1}\right) P_{\infty}^{(n)} .
\end{aligned}
$$

Proof:

i) is an immediate consequence of Lemma 3.4.

ii) For the differential $d z$ we have

$$
d z=d\left(x_{1}^{q^{2}}-x_{1}\right)=-d x_{1}
$$

and therefore for its divisor in $F_{n},(d z)^{(n)}=\left(d x_{1}\right)^{(n)}$. By [6, Remark IV.3.7.(c)]

$$
\left(d x_{1}\right)^{(n)}=-2\left(x_{1}\right)_{\infty}^{(n)}+\operatorname{Diff}\left(F_{n} / F_{1}\right)
$$

and we obtain the assertion from Proposition 3.3.
Obviously $z^{-1} d z$ is a Weil differential of $F_{n} / \mathbb{F}_{q^{2}}$ with property (3), and hence we get with (2) and Lemma 3.5 the following result for the dual codes of the codes $C_{i}, i \geq 3$ :

Theorem 3.6: For $i \geq 3$ we have

$$
C_{\mathcal{L}}\left(D^{(i)}, G^{(i)}\right)^{\perp}=C_{\mathcal{L}}\left(D^{(i)}, H^{(i)}\right)
$$

with

$H^{(i)}=\sum_{j=1}^{\lfloor(i-3) / 2\rfloor} \mu_{j} M_{2 j+1}^{(i)}+\rho E^{(i)}+\sigma P_{\infty}^{(i)}-\sum_{j=1}^{\lfloor(i-3) / 2\rfloor} D_{j}^{(i)}-\delta_{i} M_{i-1}^{(i)}$

where

$$
\begin{aligned}
\delta_{i} & = \begin{cases}1, & \text { if } i \equiv 0 \bmod 2 \\
0, & \text { if } i \boxminus 1 \bmod 2 .\end{cases} \\
\sigma & =q^{i+1}+q^{i}-q-2-s \\
\rho & =q^{i-1}+q^{i-2}-q-2-r \\
\mu_{j} & =q^{i-2 j-1}-q-2-\mu_{j} \quad \text { for } 1 \leq j \leq\left\lfloor\frac{i-3}{2}\right\rfloor .
\end{aligned}
$$

Remark 3.7: It is well known that the dual code of a Hermitian code $C_{2}$ again is a Hermitian code, namely, for $0 \leq s \leq q^{3}+q^{2}-q-2$ one has (see [6, Proposition VII.4.2])

$C_{\mathcal{L}}\left(D^{(2)}, s P_{\infty}^{(2)}\right)^{\perp}=C_{\mathcal{L}}\left(D^{(2)}, \sigma P_{\infty}^{(2)}\right)$

$$
\text { with } \sigma=q^{3}+q^{2}-q-2-s .
$$

From Theorem 3.6 we get a similar result for the codes $C_{3}$, that is

$$
C_{\mathcal{L}}\left(D^{(3)}, r E^{(3)}+s P_{\infty}^{(3)}\right)^{\perp}=C_{\mathcal{L}}\left(D^{(3)}, \rho E^{(3)}+\sigma P_{\infty}^{(3)}\right)
$$

with $\rho=q^{2}-2-r$ and $\sigma=q^{4}+q^{3}-q-2-s$.

For $i \geq 4$ it is not completely true that the dual codes are of the same type as the codes $C_{i}$, since the divisors $H^{(i)}$ prescribe in addition some zeros for the functions.

\section{THE CODES RELATED to $F_{3} / \mathbb{F}_{q^{2}}$}

Our next aim is to describe the codes corresponding to $F_{3} / \mathbb{F}_{q^{2}}$ explicitely which means that we want to determine a basis for a space $\mathcal{L}\left(G^{(3)}\right)$ and a generator matrix for $C_{3}$. Since we are only dealing with the codes related to $F_{3}$, we set

$$
\begin{gathered}
P_{\infty}:=P_{\infty}^{(3)} \quad E:=E^{(3)} \quad D_{0}:=D_{0}^{(3)} \quad D:=D^{(3)} \\
G(r, s):=G^{(3)}
\end{gathered}
$$

where

$$
0 \leq r \leq q^{2}-2 \quad 0 \leq s \leq q^{4}+q^{3}-q-2
$$

and

$$
2 q^{3}-4 q \leq(q-1) r+s<q^{4}-q^{2}+q .
$$

Then by Definition 3.2

$$
C(r, s):=C_{\mathcal{L}}(D, G(r, s))
$$

is an $[n, k, d]$ code with

$$
n=q^{4}-q^{2}+q, \quad k=(q-1) r+s-q^{3}+2 q
$$

and

$$
d \geq q^{4}-q^{2}+q-(q-1) r-s .
$$


We want to construct a basis of $\mathcal{L}(G(r, s))$ where all elements are of the form

$$
x_{1}^{i_{3}} x_{2}^{i_{2}} z_{3}^{j} \quad \text { with } i_{1}, i_{2}, j \in \mathbb{Z} \text {. }
$$

With Lemma 2.1 we get for the principal divisors of $x_{1}, x_{2}$, and $z_{3}$ in $F_{3}$

$$
\left.\begin{array}{l}
\left(x_{1}\right)=Q_{3}+D_{0}+q E-q^{2} P_{\infty} \\
\left(x_{2}\right)=q Q_{3}+q D_{0}-q E-q P_{\infty} \\
\left(z_{3}\right)=q(q+1) Q_{3}-(q+1) E-(q+1) P_{\infty}
\end{array}\right\}
$$

and from the valuations of $x_{1}, x_{2}$, and $z_{3}$ at the different places we get the conditions on the exponents $i_{1}, i_{2}, j$ such that $x_{1}^{i_{1}} x_{2}^{i_{2}} z_{3}^{j} \in \mathcal{L}(G(r, s))$. The difficult part is to find enough linearly independent elements of that form.

Definition 4.l: We define the following sets:

$$
\begin{gathered}
I_{1}(r, s):=\left\{\left(i_{1}, i_{2}, j\right) \mid 0 \leq i_{1}, 0 \leq i_{2}, j \leq q-1,\right. \\
i_{2} q+j(q+1) \leq s-i_{1} q^{2}, \\
\left.i_{2} q+j(q+1) \leq r+i_{1} q\right\} \\
I_{2}(r, s):=\left\{\left(i_{1},-i_{2}, j\right) \mid 1 \leq i_{2} \leq q, i_{1} \geq i_{2} q, 0 \leq j \leq q-1,\right. \\
j(q+1) \leq s+i_{2} q-i_{1} q^{2}, \\
j(q+1) \leq r+i_{2} q+i_{1} q, \\
\left.\left(i_{1}-1,-i_{2}+q, j\right) \notin I_{1}(r, s)\right\} \\
I_{3}(r, s):=\left\{\left(-i_{1}, i_{2}, j\right) \mid 1 \leq i_{1}, 1 \leq i_{2} \leq q-1,\right. \\
i_{2} q \geq i_{1}, 0 \leq j \leq q-1, \\
i_{2} q+j(q+1) \leq s+i_{1} q^{2}, \\
\left.i_{2} q+j(q+1) \leq r-i_{1} q\right\} \\
I(r, s):=I_{1}(r, s) \cup I_{2}(r, s) \cup I_{3}(r, s)
\end{gathered}
$$

Lemma 4.2: For $i_{1}, l_{1} \in \mathbb{Z}$ and $0 \leq i_{2}, l_{2}, j, k \leq q-1$ we have

$$
\begin{aligned}
i_{1} q^{2}+i_{2} q+j(q+1)=l_{1} q^{2}+l_{2} q+ & k(q+1) \\
& \Leftrightarrow\left(i_{1}, i_{2}, j\right)=\left(l_{1}, l_{2}, k\right) .
\end{aligned}
$$

Proof: Trivial.

Theorem 4.3: The set

$$
B(r, s):=\left\{x_{1}^{i_{1}} x_{2}^{i_{2}} z_{3}^{j} \mid\left(i_{1}, i_{2}, j\right) \in I(r, s)\right\}
$$

is a basis of $\mathcal{L}(G(r, s))$ over $\mathbb{F}_{q^{2}}$.

Proof: Using (5) and Definition 4.1 one can easily verify that

$$
\left(x_{1}^{i_{1}} x_{2}^{i_{2}} z_{3}^{j}\right) \geq-G(r, s) \text { for }\left(i_{1}, i_{2}, j\right) \in I(r, s)
$$

which means that $B(r, s) \subseteq \mathcal{L}(G(r, s))$.

Let $u=x_{1}^{i_{1}} x_{2}^{i_{2}} z_{3}^{j} \in B(\bar{r}, s)$. Then

$$
-v_{P_{\infty}}(u)=i_{1} q^{2}+i_{2} q+j(q+1)
$$

and from Lemma 4.2 we obtain that all elements in $B(r, s)$ have different orders at $P_{\infty}$, which implies that they are linearly independent. (Observe that for $\left(i_{1}, i_{2}, j\right) \in I_{2}(r, s)$ we have $\left(i_{1}-1, i_{2}+q, j\right) \notin I_{1}(r, s)$ and $i_{1} q^{2}+i_{2} q+j(q+1)=$ $\left.\left(i_{1}-1\right) q^{2}+\left(i_{2}+q\right) q^{2}+j(q+1).\right)$

Since the dimension of $C(r, s)$ is $k=(q-1) r+s-q^{3}+2 q$ (see (4)) it remains to prove that $\# I(r, s)=k$. In order to count the elements of $I(r, s)$ we need some preparations.
Definition 4.4: For $l \in \mathbb{Z}$ we define

i) $h(l):=\#\{(i, j) \mid 0 \leq i, 0 \leq j \leq q-1$,

$$
i q+j(q+1) \leq l\}
$$

ii) $\mu(l):= \begin{cases}0, & \text { if } l \leq 0 \\ \left\lfloor\frac{l}{q+1}\right\rfloor+1, & \text { if } 0 \leq l<q^{2}-1 \\ q, & \text { if } l \geq q^{2}-1 .\end{cases}$

For $l \geq q^{2}-q-1$ we have (see $[6$, p. 212)

$$
h(l)=l+1-\frac{1}{2} q(q-1)
$$

and it is easy to check that for $l \geq 0$

$$
h(l)+\mu(l+q)=h(l+q) .
$$

Now we set (for $r$ and $s$ as usual)

$$
a:=\left\lfloor\frac{s-r}{q(q+1)}\right\rfloor \quad d:=\left\lfloor\frac{a}{q}\right\rfloor \quad \text { and } \quad c:=\left\lfloor\frac{s}{q\left(q^{2}-1\right)}\right\rfloor \text {. }
$$

Lemma 4.5:

i) $c-1 \leq d \leq c$, if $c<q$ or $r \neq 1$.

ii) $d=q-2$ if $c=q$ and $r=1$.

iii) If $d=0$ then

$$
\begin{array}{lll}
a=q-1 & \text { and } & r \geq q^{2}-q-3, \text { or } \\
a=q-2 & \text { and } & r=q^{2}-2, \text { or } \\
c=q=2 & \text { and } & a=r=1 .
\end{array}
$$

Proof: We write $s=c q\left(q^{2}-1\right)+\beta$ with $0 \leq \beta<q^{3}-q$. Recall that

$$
2 q^{3}-4 q<(q-1) r+s<q^{4}-q^{2}+q .
$$

From this follows that $1 \leq c \leq q$, and if $c=q$ then $r=0$ and $\beta<q$, or $r=1$ and $\beta=0$. If $c<q$, then

$$
((q-1) / q) c-1 / q \leq a / q \leq((q-1) / q)(c+1)
$$

thus $c-1 \leq d \leq c$. i) and ii) now follow immediately.

Suppose $d=0$. i) and ii) yield either $c=2=q$ and $r=1=a$ or $c=1$. For $c=1$ we have

$$
a=q-1+\lfloor(\beta-r) / q(q+1)\rfloor
$$

and

$$
s=q^{3}-q+\beta>2 q^{3}-4 q-(q-1) r
$$

that implies

$a>q-2+\left(q^{2}-3-r\right) /(q+1)=2 q-3-(r+2) /(q+1) \geq q-2$

and since $d=0$ also $a \leq q-1$. If $a=q-1$, then

$$
q^{2}+q>\beta-r>q^{3}-3 q-q r
$$

hence $r \geq q^{2}-q-3$, and if $a=q-2$, then

$$
0>\beta-r>q^{3}-3 q-q r
$$

hence $r=q^{2}-2$. 
Definition 4.6: We define the set

$$
\begin{gathered}
J(r, s):=\left\{\left(i_{1},-i_{2}, j\right) \mid 1 \leq i_{2} \leq q, i_{1} \geq i_{2} q, 0 \leq j \leq q-1,\right. \\
j(q+1) \leq s+i_{2} q-i_{1} q^{2}, \\
\left.j(q+1) \leq r+i_{2} q+i_{1} q\right\} .
\end{gathered}
$$

The following remark is easy to check.

Remark 4.7:

i) $I_{2}(r, s)=J(r, s) \backslash J\left(r-q^{2}-q, s\right)$

ii) $r+i q \leq s-i q^{2} \Leftrightarrow i \leq a$

iii) $h(r-q)=h\left(r-\left\lfloor\frac{r}{q}\right\rfloor q\right)$

$$
+\sum_{i=1}^{\lfloor r / q\rfloor-1} \mu(r-i q) \text { for } \mathrm{r} \geq 2 q \text {. }
$$

With Remark 4.7 ii) and Definition 4.4 i) we obtain

$$
\begin{aligned}
\# I_{1}(r, s)= & \sum_{i=0}^{a}\left(h(r+i q)-h\left(r-q^{2}+i q\right)\right) \\
& +\sum_{i=a+1}^{\left\lfloor s / q^{2}\right\rfloor}\left(h\left(s-i q^{2}\right)-h\left(s-(i+1) q^{2}\right)\right) \\
= & h\left(s-(a+1) q^{2}\right)+\sum_{i=0}^{a}(h(r+i q) \\
& \left.-h\left(r-q^{2}+i q\right)\right) .
\end{aligned}
$$

(Observe that in the definition of $I_{1}(r, s)$ we have $i_{2} \leq q-1$.)

By Remark 4.7 i), ii) and Definition 4.4 ii) follows

$$
\begin{aligned}
& \# I_{2}(r, s)=\sum_{i_{2}=1}^{d}\left(\sum_{i_{1}=i_{2} q}^{a} \mu\left(r+i_{2} q+i_{1} q\right)\right. \\
&\left.\quad-\sum_{i_{1}=i_{2} q}^{a+1} \mu\left(r-\left(q^{2}+q\right)+i_{2} q+i_{1} q\right)\right) \\
&+\sum_{i_{2}=1}^{d}\left(\sum_{i_{1}=a+1}^{\left\lfloor\left(s+i_{2} q\right) / q^{2}\right\rfloor} \mu\left(s+i_{2} q-i_{1} q^{2}\right)\right. \\
&\left.-\sum_{i_{1}=a+2}^{\left\lfloor\left(s+i_{2} q\right) / q^{2}\right\rfloor} \mu\left(s+i_{2} q-i_{1} q^{2}\right)\right)+\epsilon(a)
\end{aligned}
$$

where

$$
\epsilon(a)= \begin{cases}\mu\left(s-(d+1) q\left(q^{2}-1\right)\right)-\mu\left(r+d\left(q^{2}+q\right)\right) \\ \text { if } d<c \text { and } a \equiv-1 \bmod q \\ \text { else. }\end{cases}
$$

Thus by (7)

$$
\begin{aligned}
\# I_{2}(r, s)= & h\left(s+d q-(a+1) q^{2}\right)-h\left(s-(a+1) q^{2}\right) \\
& +\epsilon(a)-h\left(r-q+d\left(q^{2}+q\right)\right) \\
& +h(r-q)+\sum_{i=1}^{d}(h(r+a q+i q) \\
& \left.-h\left(r-q^{2}+a q+i q\right)\right) .
\end{aligned}
$$

Finally, from Remark 4.7 iii) we get

$$
\# I_{3}(r, s)=\sum_{i=2}^{\lfloor r / q\rfloor} h(r-i q)
$$

Lemma 4.8: For $d \geq 1$ we have

$$
s+d q-(a+1) q^{2} \geq q^{2}-q-1
$$

and

$$
r-q^{2}+q+a q+d q \geq q^{2}-q-1
$$

Proof: We write again $s=c q\left(q^{2}-1\right)+\beta$ with $0 \leq$ $\beta<q^{3}-q$. As $d \geq 1$ and $(q-1) r+s>2 q^{3}-4 q$ we have

$$
\begin{aligned}
(q+1) & \left(2 q^{2}-q-1\right) \\
= & 2 q^{3}+q^{2}-2 q-1<q r+s+d\left(q^{2}+q\right)+q-1 \\
= & (q+1) s+d\left(q^{2}+q\right)-q\left(c q\left(q^{2}-1\right)\right. \\
& +\beta-r)+q-1 .
\end{aligned}
$$

Therefore,

$$
\begin{aligned}
2 q^{2}-q-1 & \leq s+d q-q^{2}\left(c(q-1)+\frac{\beta-r}{q(q+1)}\right) \\
& \leq s+d q-a q^{2}
\end{aligned}
$$

and hence

$$
s+d q-(a+1) q^{2} \geq q^{2}-q-1
$$

Moreover,

$$
\begin{aligned}
& (q+1)\left(2 q^{2}-2 q-1\right) \\
& \quad=2 q^{3}-3 q-1<q r+s+(d-1)\left(q^{2}+q\right)+q-1
\end{aligned}
$$

thus

$$
\begin{aligned}
2 q^{2}-2 q-1 & \leq r+c q(q-1)+\frac{\beta-r}{q+1}+(d-1) q \\
& \leq r+a q+d q
\end{aligned}
$$

which implies

$$
r-q^{2}+q+a q+d q \geq q^{2}-q-1 .
$$

The next proposition finishes the proof of Theorem 4.3. Proposition 4.9:

$$
\# I(r, s)=(q-1) r+s-q^{3}+2 q .
$$

Proof: First we consider the case $d \geq 1$. Using (8)-(10) we find

$$
\begin{aligned}
\# I(r, s)= & h\left(s+d q-(a+1) q^{2}\right)+\epsilon(a)-h\left(r-q+d\left(q^{2}+q\right)\right) \\
& +\sum_{i=0}^{d+a}\left(h(r+i q)-h\left(r-q^{2}+i q\right)\right)+\sum_{i=1}^{\lfloor r / q\rfloor} h(r-i q) \\
= & h\left(s+d q-(a+1) q^{2}\right)+\epsilon(a)-h\left(r-q^{2}+d\left(q^{2}+q\right)\right) \\
& +\sum_{i=1}^{q} h\left(r-q^{2}+d q+a q+i q\right)
\end{aligned}
$$

For $c>d$ and $a \equiv-1 \bmod q$ it is easy to verify by (7) that $h\left(s+d q-(a+1) q^{2}\right)+\epsilon(a)$

$$
=h\left(s+q+d q-(a+1) q^{2}\right)-q .
$$


The assertion for $d \geq 1$ is now an immediate consequence of (11), (6), and Lemma 4.8. Let now $d=0$. Using (8)-(10), Lemma 4.5 iii), and (6) we obtain

$$
\begin{aligned}
\# I(r, s)= & h\left(s-(a+1) q^{2}\right)+\epsilon(a)+\sum_{i=0}^{a} h(r+i q) \\
& -\sum_{i=q-a}^{q} h(r-i q)+\sum_{i=2}^{\lfloor r / q\rfloor} h(r-i q) \\
= & \left\{\begin{array}{c}
h\left(s-q^{3}+q\right)+\sum_{i=1}^{q-1} h(r+i q) \\
\text { if } a=q-1 \\
h\left(s-q^{3}+q^{2}\right)+\sum_{i=0}^{q-2} h(r+i q) \\
\text { if } a=q-2 \text { and } c=1 \\
h(4)+1+h(1)+h(3) \\
\text { if } c=q=2
\end{array}\right. \\
= & (q-1) r+s-q^{3}+2 q .
\end{aligned}
$$

Corollary 4.10: The pole numbes of $P_{\infty}$ in $F_{3}$ are of the form

$$
i_{1} q^{2}+i_{2} q+j(q+1) \text { with }\left(i_{1}, i_{2}, j\right) \in \bigcup_{s \geq 0} I(0, s) .
$$

Example 4.11: It is well known that the pole numbers of $P_{\infty}$ in the Hermitian function field are generated by $q$ and $q+1$, which implies that the set generated by $q^{2}$ and $q(q+1)$ is a subset of the pole numbers of $P_{\infty}$ in $F_{3}$. One would perhaps guess that there is just one other generator needed to get the whole set, but that is not true as the following examples show.

For $q=2$ the generators are: $4,6,9,11$.

For $q=3$ the generators are: $9,12,22,28,32,35$.

For $q=4$ the generators are: $16,20,37,58,65,70,75,79$.

Our next aim is to specify a generator matrix for the codes $C(r, s)$. First we introduce some new notations. We define for $\alpha \in \mathbb{F}_{q^{2}} \backslash\{0\}$ the set

$$
\begin{aligned}
M_{\alpha}:=\left\{(\beta, \gamma) \in\left(\mathbb{F}_{q^{2}}\right)^{2} \mid \beta^{q}+\beta=\right. & \alpha^{q+1} \\
& \text { and } \left.\gamma^{q}+\gamma=\left(\alpha^{-1} \beta\right)^{q+1}\right\} .
\end{aligned}
$$

For $\alpha \in \mathbb{F}_{q^{2}} \backslash\{0\}$ and $(\beta, \gamma) \in M_{\alpha}$ let $P_{\alpha \beta \gamma} \in \mathbb{P}\left(F_{3}\right)$ be the common zero of $x_{1}-\alpha, z_{2}-\beta$, and $z_{3}-\gamma$. From the equations of the function fields $F_{2}$ and $F_{3}$ over $F_{q^{2}}$ follows obviously that such places exist and that for $\alpha \in \mathbb{F}_{q^{2}} \backslash\{0\}$

$$
\operatorname{con}_{F_{3} / F_{1}}\left(P_{\alpha}\right)=\sum_{(\beta, \gamma) \in M_{\alpha}} P_{\alpha \beta \gamma}
$$

We define moreover $M_{0}:=\left\{\epsilon \in \mathbb{F}_{q^{2}} \mid \epsilon^{q}+\epsilon=0\right\}$ and $P_{00 \epsilon} \in \mathbb{P}\left(F_{3}\right)$ the common zero of $x_{1}, x_{2}$, and $z_{3}-\epsilon$ for $\epsilon \in M_{0}$. Now we can rewrite the divisor $D$ as

$$
D=\sum_{\epsilon \in M_{0}} P_{00 \epsilon}+\sum_{\alpha \in F_{q^{2}} \backslash\{0\}} \sum_{(\beta, \gamma) \in M_{\alpha}} P_{\alpha \beta \gamma} .
$$

Next we fix some ordering on the set

$$
M:=\left\{(\alpha, \beta, \gamma) \mid P_{\alpha \beta \gamma} \leq D\right\}
$$

and define for $m=i_{1} q^{2}+i_{2} q+j(q+1)$ with $i_{1}, i_{2}, j \in \mathbb{Z}$ the vector

$$
u_{m}:=\left(u_{\alpha \beta \gamma}\right)_{(\alpha, \beta, \gamma) \in M}
$$

where

$$
u_{\alpha \beta \gamma}:= \begin{cases}\alpha^{i_{1}-i_{2}} \beta^{i_{2}} \gamma^{j}, & \text { if } \alpha \neq 0 \\ 0, & \text { if } \alpha=0 \text { and }\left(i_{1} \neq 0 \text { or } i_{2} \neq 0\right) \\ \gamma^{j}, & \text { if } \alpha=0 \text { and } i_{1}=i_{2}=0 .\end{cases}
$$

Corollary 4.12: Let

$$
\left\{m_{l}\right\}_{l=1}^{k}=\left\{i_{1} q^{2}+i_{2} q+j(q+1) \mid\left(i_{1}, i_{2}, j\right) \in I(r, s)\right\}
$$

with $m_{l}<m_{l+1}$ for $1 \leq l \leq k-1$. Then the $k \times\left(q^{4}-q^{2}+q\right)$ matrix whose rows are $u_{m_{1}}, \cdots, u_{m_{k}}$ is a generator matrix of $C(r, s)$.

Proof: This is an immediate consequence of Theorem 4.3 and the fact, that for $u=x_{1}^{i_{1}} x_{2}^{i_{2}} z_{3}^{j} \in B(r, s)$ we have $u\left(P_{\alpha \beta \gamma}\right)=u_{\alpha \beta \gamma}$.

The codes from $F_{3}$ considered here are better than $\mathrm{BCH}$ codes, and are comparable with the codes coming from the function field studied by Petersen and Sørensen in [5]. These codes over $F_{q^{2}}$ have $n=q^{4}$ and

$$
k+d \geq q^{4}-\frac{1}{2} q^{3}+\frac{1}{2} q+1
$$

where the codes we consider have $n=q^{4}-q^{2}+q$ and $k+d \geq q^{4}-q^{3}-q^{2}+3 q$.

Finally, we give an example showing that one cannot find analogous bases for the spaces $\mathcal{L}\left(G^{(i)}\right)$ with $i \geq 4$. By an analogous basis we mean a set of linearly independent functions of the form

$$
f=\prod_{l=1}^{i-1} x_{l}^{i_{l}} z_{i}^{j} \in \mathcal{L}\left(G^{(i)}\right) .
$$

Example 4.13: We consider the function field $F_{4} / F_{4}$. Its genus is $g_{4}=13$ and the pole numbers of the functions $f \in \mathcal{L}\left(24 P_{\infty}^{(4)}\right)$ that are of the form (12) are

$$
0,8,12,16,18,20,22,23,24 \text {. }
$$

From the Weierstrass Gap Theorem (see [6, Theorem I.6.7]) we see that three pole numbers are missing.

Remark 4.14: Already in this simple example we see, that the functions of the type (12) only generate a subspace of $\mathcal{L}\left(G^{(i)}\right)$ for $i \geq 4$. An idea could be to consider sequences of subcodes of the codes $C_{i}$ in Definition 3.2 replacing the spaces $\mathcal{L}\left(G^{(i)}\right)$ by the largest subspaces generated by functions as in (12). However, after computing many examples of such subcodes in $F_{4}$ and $F_{5}$, to us such an attempt appears not very promising, since we got the impression that those subcodes are asymptotically bad.

\section{REFERENCES}

[1] V. G. Drinfeld and S. G.Vlădut,, "Number of points of an algebraic curve" Func. Anal., vol. 17, pp. 53-54, 1983.

[2] A. Garcia and H. Stichtenoth, "A tower of Artin-Schreier extensions of function fields attaining the Drinfeld-Vlädut bound," Inventiones Math., vol. 121 , pp. $211-222,1995$.

[3] Y. Ihara, "Some remark on the number of rational points of algebraic curves over finite fields," J. Fac. Sci. Tokyo, vol. 28, pp. 721-724, 1981. 
[4] G. L. Katsman, M. A. Tsfasman, and S. G. Vlăđut,", Modular curves and codes with a polynomial construction," IEEE-Trans. Inform. Theory, vol. IT-30, no. 2, pp. 353-355, Mar. 1984.

[5] J. P. Pedersen and A. B. Sørensen," "Codes from certain algebraic function fields with many rational places," MAT-Rep. 1990-11, Mathematical Institute, The Technical University of Denmark, Lyngby, 1990.

[6] H. Stichtenoth, Algebraic Function Fields and Codes (Springer Universitext). Berlin-Heidelberg-New York: Springer, 1993.
[7] M. A. Tsfasman and S. G. Vlădut,Algebraic-Geometric Codes. Dordrecht-Boston-London: Kluwer, 1991.

[8] M. A. Tsfasman, S. G. Vlădut, and T. Zink, "Modular curves, Shimura curves and Goppa codes, better than the Varshamov-Gilbert bound," Math. Nachr., vol. 109, pp. 21-28, 1982.

[9] S. G. Vlădut and Y. I. Manin, "Linear codes and modular curves, $J$. Sov. Math., vol. 30, pp. 2611-2643, 1985.

[10] G. L. Feng and T. R. N. Rao, "Improved geometric Goppa codes, part II. Generalized Klein codes," preprint. 